\title{
Research on Distributed Signal Processing Technology in Wireless Sensor Networks
}

\author{
WU Zhengnan ${ }^{1, a}$, CHENG Yuansheng ${ }^{2}$, LU Jun ${ }^{1}$ \\ ${ }^{1}$ School of Electronics and Information Engineering, Wuhan Donghu University, HuBei Wuhan, \\ 430212, China \\ ${ }^{2}$ The Hydrological and Water Resources Survey Bureau of The Middle ChangJiang River, \\ ChangJiang Water Resources Commission, HuBei Wuhan, 430010, China \\ a foxwzn0716@163.com
}

Keywords: Distributed Networks, Cognitive Radio Sensor Networks, Mobile Networks, Networked Signal Processing,

\begin{abstract}
Wireless sensor network is widely used in military, industrial, medical, transportation and other fields. As a product of the era in the future, wireless sensor network will play a more and more important role. This network is usually composed of sensor nodes that of low power consumption and low cost. Data source node is responsible for the collection and detection range, and multiple hops to pass data gathering node by way of relay node. However, because the sensor nodes in wireless sensor networks mainly limited through energy battery power supply, and the limited channel capacity limits the data rate in the whole network of transmission and distribution, it is a key issue that how to ensure the quality of service of wireless sensor network through effective allocation of network resources and queue scheduling.
\end{abstract}

\section{Wireless sensor network}

Relative to the wireless network of the infrastructure, wireless networks that of no infrastructure has many unique advantages that it can go deep into the various and unknown environment, thus obtained the swift and violent development in recent years. So far, some relatively mature wireless network technology is more and deeper into every aspect of social life. At the same time, along with the wireless communication technology, embedded computing technology, information processing technology and the rapid development of sensor technology, some emerging, wireless network technology that needs further research emerged, such as wireless sensor networks, wireless Ad hoc networks and wireless Mesh networks. [1]Wireless sensor network (WSN) that set data acquisition, processing, transmission, communication as one has especially attracted a great deal of attention, which is becoming a hot spot of current research.

\section{Characteristics of the wireless sensor network}

As a new generation of effective access to information of wireless network, wireless sensor network (WSN) is made up of many sensor nodes deployed in monitoring area by self-organizing network system, the main purpose of their application is coverage area of perception object information through collaborative perception, collection and processing network and send it to the external users. In the entire network, sensor nodes with awareness and communication ability is the power from the power supply, perception, and memory processor unit and the corresponding communication components and software and so on several parts, general sensor nodes as shown in figure below. 


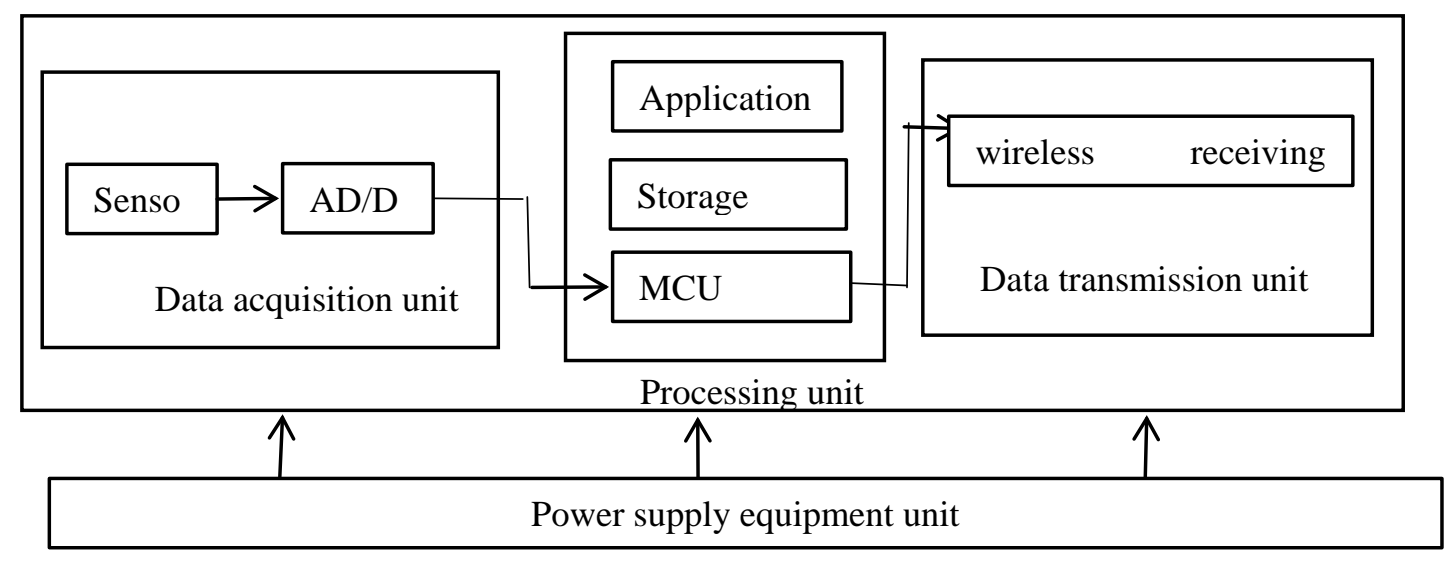

Figure1 Sensor nodes structure diagram

\section{Constraints facing wireless sensor network applications}

Although there have been widely studies in wireless sensor network (WSN), there are also some corresponding network into practice, but in terms of the current technical level, the normal operation of wireless sensor network security practical use also faces many problems, main factors that limit the application of the summary is as follows:

Cost: the cost of sensor nodes is an important factor that restricts its mass widely used. It is required to balance cost time, data accuracy and energy supply based on the specific application requirements.

Energy consumption: most applications need network using disposable independent power supply system, therefore requires work to low energy consumption, prolong the network life cycle, this is one of the important factors to expand application.

Miniaturization: in some areas, the volume of a node need to be miniaturization, have no effect on the target itself, or not to be found to accomplish particular task.

Positioning performance: the target positioning accuracy and hardware resources, network scale, the environment around, the anchor nodes is related, target positioning technology is one of the hot spot of current research.

Mobility: in some specific applications, the node or gateway need to move, causing difficulties in the network rapidly since the group, the factor is also one of the major problems affecting its application.

\section{Distributed processing}

From the perspective of data processing, line sensor network is described as a distributed database system composed of a large number of sensor nodes of different function data-centric structures. In the network according to the regulation of routing protocols and operators, each sensor node has a relatively clear division of labor, acquisition node is responsible for the perception and gathers information on monitoring area, and sorting and calculating acquisition processing node information collected, access user interface node is provided to the user the way of dealing with data. [2]The user can collect in the whole sensor network through the node manually process the information.

\section{Distributed data compression}

A large amount of data transmission is needed in sensor networks. Through compress the data transmission way sensor nodes can reduce the energy consumption of sensors in the network communication. Centralized processing network complete compression in a node set while distributed processing network compression task was assigned to multiple nodes together complete, compressed data sent through multiple hops way to the node to unpack again after treatment.

\section{Distributed data management}

In the traditional database system, the centralized approach is generally used. If use the traditional way of database architecture in the wireless sensor network, it can make sensor nodes 
responsible for the centralized storage management data overweight, and a single sensor node can bear a large amount of data needed for the center server storage tasks. So database architecture based on of distributed processing system is often used in wireless sensor networks. In the large-scale network, each node only store part of the data, each node can initiate, forwarding and response data query command. By means of this kind of distributed architecture, the way of centralized architecture center server task is balanced to multiple nodes, which can effectively balance the energy consumption of the entire network. [3]The key components of distributed database architecture is to solve how to improve the response speed of query command, sensor nodes in the network to efficiently store and transmit data, problems such as how to build a more efficient indexing algorithm.

\section{Distributed image compression}

In wireless sensor network, the energy is limited by great sensor nodes in the storage capacity and computing ability, communication ability, etc, also has greatly restricted. So in the wireless sensor network and data collection and transmission, it is not suitable to use the collected data in the case of no compression for direct transmission.

Wireless sensor network node layout is usually relatively dense, high redundancy of information between nodes, a great amount of data of untreated information transmission will increase energy consumption in node communication, waste a precious network bandwidth, the communication link congestion, reduce the efficiency of data collection and transmission, and reduce the transmission of timeliness, increase the complexity of the transmission, reducing the life of sensor nodes. So distributed image compression technology in wireless sensor network transmission of multimedia information, such as large amount of data becomes particularly important.

\section{Cognitive distributed signal processing technology of sensor network research}

\section{Power consumption model of the network}

Cognitive power consumption of the sensor network consists of three parts: multitasking perception, signal transmission and data transmission. This article assumes that there are $\mathrm{N}$ cognitive sensor nodes and a total of $\mathrm{K}$ clusters. The cluster at number $k$ is marked as $c_{k}$, and having $N_{k}$ cognitive sensor nodes. Clusters $c_{k}$ of the $i$ th node is remembered as $n_{i}^{k}$, the coordinates of $\left(x_{i}, y_{i}\right)$.

Data transmission includes communications within the cluster and the cluster communication. Within the cluster communication stage, all the cognitive sensor nodes through mutual available channel to report back to the cluster head their sensing results. In the cluster number $k$, when the first $j$ was chosen for cluster head nodes, the power consumption of cluster communication is the $n$ the total for all the members of cluster.

$$
P_{\text {intra }}\left(i_{k}=j\right)=\sum_{\substack{i=1 \\ i \neq j}}^{N_{k}} P_{i}\left(n_{i}^{k}\right)
$$

In this paper, all the cluster members automatically adjust the transmission power according to the SNR requirement received to save energy. This can be done through the following ways: first, estimate the cluster head to the members of cluster according to the received signal strength channel fading, and then calculate how strong need to use the transmission power according to the right channel model. Because the distance between the cluster members and their cluster heads are usually relatively close, spatial channel model with free can be applied[4].

$$
P_{t}\left(n_{i}^{k}\right)=C_{0} \cdot P_{r} \cdot d^{2}\left(n_{i}^{k}, n_{j}^{k}\right)
$$

$P_{r}$ is the minimum power at the receiving end in order to make the decoding correctly. $C_{0}$ is constant related to the attenuation coefficient.

Assume that when the first $j$ nodes were selected as cluster heads, power consumption within the cluster can be rewritten as: 


$$
P_{\text {int ra }}\left(C H=n_{j}^{k}\right)=\sum_{\substack{i=1 \\ i \neq j}}^{N_{k}} P_{t}\left(n_{j}^{k}\right)=C_{0} P_{r} \sum_{\substack{i=1 \\ i \neq j}}^{N_{k}} d^{2}\left(n_{i}^{k}, n_{j}^{k}\right)
$$

In order to balance power consumption, cognitive sensor nodes often become cluster heads in turn. The probability of the first $j$ a node to become cluster head is $p\left(C H=\mathrm{n}_{j}^{k}\right)$. From statistical point of view, power consumption of cluster $c_{k}$ total within the cluster is:

$$
\begin{aligned}
& P_{\text {intra }}\left(c_{k}\right)=\sum_{\substack{i=1 \\
i \neq j}}^{N_{k}} P\left(C H=n_{j}^{k}\right) P_{\text {intra }} P\left(C H=n_{j}^{k}\right) \\
& =C_{0} P_{r} \sum_{j=1}^{N_{k}} p\left(C H=n_{j}^{k}\right) \sum_{\substack{i=1 \\
i \neq j}}^{N_{k}} d^{2}\left(n_{i}^{k}, n_{j}^{k}\right)
\end{aligned}
$$

After receiving the sensing information of all the cluster members, cluster heads usually compressed information first, then transfer the sensing information to the upstream node with a common channel by way of relay, and ultimately converge to convergent node, which is referred to as a cluster of communication between phases. Therefore, cluster of communication between the total power consumption can be expressed as:

$$
P_{\text {inter }}=\sum_{k=1}^{K} P_{I C}=K C_{0} P_{R} d_{\max }^{2}
$$

\section{The optimal number of clusters}

There are a total of $\mathrm{N}$ cognitive sensor nodes in this paper and hope to put them into K cluster. How many clusters will be divided is the key of energy saving. Expectations for the power consumption of data transmission are:

$$
E\left(P_{D A T A}\right)=2 C_{0} P_{r} \cdot E\left(\sum_{k=1}^{K} \sum_{i=1}^{N_{k}} d^{2}\left(n_{i}^{k}, \operatorname{center}(k)\right)\right)+K \cdot C_{0} P_{r} \cdot d_{\max }^{2}
$$

It is reasonable to assume that random and cognitive sensor nodes are evenly distributed in a two-dimensional space around the center point, and density $\rho$ is presupposed by sensing application related requirements. Therefore:

$$
E\left(\sum_{k=1}^{K} \sum_{i=1}^{N_{k}} d^{2}\left(n_{i}^{k}, \text { center }(k)\right)\right)=N\left(\operatorname{var}\left(x_{i}^{k}\right)+\operatorname{var}\left(y_{i}^{k}\right)\right)=\frac{N d^{2}}{6}
$$

Take the type into type (2.9), it can be:

$$
E\left(P_{\text {DATA }}\right)=C_{0} P_{r}\left(\frac{N^{2}}{3 \rho K}+K d_{\max }^{2}\right)
$$

\section{Energy saving box optimization}

After determine the optimal number of clusters $K_{\text {opt }}$, this paper found that from the perspective of clustering, the entire network energy consumption was mainly affected by communications within the cluster of energy. According to the type (2.7), therefore, to minimize the entire network is equivalent to minimize power consumption cognitive sensor nodes to their sum of squares of the cluster center distance, namely:

$$
\min E_{\text {network }} \Leftrightarrow \min \sum_{k=1}^{K} \sum_{i=1}^{N_{k}} d^{2}\left(n_{i}^{k}, \text { center }(k)\right)
$$

\section{Summary}

Distributed on a large scale network is one of the important direction of the future evolution of the wireless network, its distributed characteristics may bring revolutionary change to the existing network structure and algorithm, thus bring many frontier theoretical problems and challenges. This dissertation is to future distributed object couplet network as the background, aiming at high 
cognitive sensor network, mobile network of two kinds of concrete forms, some of the basic theory and key technologies, such as distributed signal processing technology, multiple access channel distributed cognition, and carried on the thorough analysis and research on distributed information transmission theory, and some meaningful conclusions are obtained, for the future of distributed network application and popularization has a certain theoretical value and practical significance.

\section{Acknowledgements}

Wuhan Donghu University"Communication and Information Engineering" Hubei province key experimental teaching demonstration center project.

\section{References}

[1] M. Grossglauser and D. Tse. Mobility increases the capacity of ad hoc wireless networks. IEEE/ACM Trans. Networking,10(4):477-486, Aug. 2002.

[2] Special issue on collaborative signal and information processing in micro-sensor networks. IEEE Signal Processing Magazines, Mar. 2002.

[3] P. Dragotti M. Gastpar and M. Vetterli. The distributed, partial, and conditional karhunen-loeve transforms. Data Compression Conf., pages 283-292, Mar. 2003.

[4] R. Neelamani V. Delouille and R. Baraniuk. Robust distributed estimation in sensor networks using the embedded polygons algorithm. Proc. 3rd Int. Symp. Information Processing in Sensor Networks, Apr.2004. 